\title{
DELINEAMENTO EM BLOCOS CASUALIZADOS COMPLETOS AUMENTADOS*
}

MARIA CRISTINA STOLF NOGUEIRA** F. PIMENTEL GOMES $* \star *$

\section{RESUMO}

O objetivo do presente trabalho consistiu em apresentar uma metodologia mais simples para a realização da anālise de variân cia de experimentos em blocos casualizados completos aumentados.

\section{INTRODUÇÃO}

Delineamento aumentado é um tipo de delineamen to experimental formado a partir de qualquer deli= neamento, pelo acréscimo de parcelas nos blocos. Foi introduzido por FEDERER (1956), no Havaí, com - objetivo de solucionar problemas encontrados ao comparar plântulas ("seedlings") e "variedades" (que melhor se chamariam clones) de cana-de-açúcar,

* Seminário apresentado durante o Curso de Pós-Graduação em Experimentação e Estatística da Escola Superior de Agricultura "Luiz de Queiroz", USP, Piracicaba,SP. Entregue para publicação em 23.11.78.

** Departamento de Matemática e Estatística, E.S.A. "Luiz de Queiroz", USP.

*** Departamento de Matemātica e Estatística, E.S.A. "Luiz de Queiroz", USP. 
abacaxi, etc., no decorrer do desenvolvimento de um programa de melhoramento. Basicamente, os deli neamentos aumentados para blocos casualizados com= pletos e quadrados latinos, apresentam um conjunto de tratamentos comuns, repetido b vezes, e um segundo grupo de tratamentos, denominados tratamentos regulares, que aparecem uma única vez. Este de lineamento possui uma gama de aplicação muito ampla, podendo ser utilizado em todos os campos onde se desejam combinar, num mesmo experimento, cultivares novos já selecionados com cultivares promissores.

Uma análise intrablocos de um grupo de experimentos em blocos casualizados completos, onde alguns tratamentos são comuns para todos os grupos, foi proposta por PIMENTEL GOMES \& GUIMARÃES (1958). Estes tratamentos foram considerados como tratamen tos comuns, e os demais, específicos para cada gru po, foram denominados tratamentos regulares. A ană lise do delineamento estudado é um caso especial de blocos incompletos equilibrados intra e inter grupos. O conjunto dos experimentos, considerado como um delineamento usual em blocos incompletos, foi analisado conjuntamente, admitindo-se que apre sentassem variâncias semelhantes.

Comparando os trabalhos desenvolvidos por FEDE RER (1956) e por PIMENTEL GOMES \& GUIMAR ÃES (1958), observa-se que há uma certa semelhança entre eles, onde cada bloco do delineamento aumentado corres ponde a um experimento em blocos casualizados com pletos, considerado sem repetição.

A finalidade deste trabalho, è apresentar uma metodologia mais simples para a realização da anáIise de variância de experimentos em blocos casualizados completos aumentados. 


\section{- ESQUEMA DA ANÁLISE DE VARIANCIA}

Suponhamos um experimento em blocos casualizados completos aumentados, com as seguintes caracte risticas:

$$
t=c+2,
$$

sendo que: $t=$ número total de tratamentos;

$c=$ numero de tratamentos comuns $-y_{i j}$

$(i=1, \ldots, c)$,

$(j=1, \ldots, b)$;

$z=$ número de tratamentos regulares $-y_{j g}$ $\left(\begin{array}{l}j=1, \ldots, b) \\ \left(g=1, \ldots, r_{j}\right) .\end{array}\right.$

$\mathrm{b}=$ número de blocos;

$r_{j}=$ número de tratamentos regulares por bloco, portanto,

$\sum r_{j}=z_{i}$

$\mathrm{N}_{j}=$ número de parcelas por bloco, portanto,

$N_{j}=c+r_{j} ;$

$\mathrm{N}$ = número total de parcelas, portanto,

$$
N=b \cdot c+z=b \cdot c+\sum r_{j}
$$

\section{- CAsualização}

Há b blocos com $N_{j}$ parcelas $(j=1, \ldots, b)$. A casualização é efetuada da seguinte maneira: em primeiro lugar, sorteiam-se os $c$ tratamentos comuns entre as $\mathrm{N}_{j}$ parcelas de cad̄a bloco, e, em seguida, sorteiam-se os $z$ tratamentos regulares entre as parcelas restantes de cada bloco. 
Tabela 1 - Representação simbólica dos dados em delineamento de blocos casualizados completos aumentados.

\begin{tabular}{|c|c|c|c|c|c|c|}
\hline \multirow{2}{*}{$\begin{array}{l}\text { Tratamentos } \\
\text { comuns }\end{array}$} & \multicolumn{4}{|c|}{ Blocos } & \multirow{2}{*}{ Total } & \multirow{2}{*}{ Média } \\
\hline & 1 & 2 & $\ldots$ & b & & \\
\hline 1 & $y_{11}$ & $\mathrm{y}_{12}$ & $\cdots$ & $y_{1 b}$ & $y_{1}$ & $\bar{y}_{1}$ \\
\hline 2 & $\mathrm{y}_{21}$ & $\mathrm{y}_{22}$ & $\cdots$ & $\mathrm{y}_{2 \mathrm{~b}}$ & $y_{2}$ & $\bar{y}_{2}$ \\
\hline - & $\cdots$ & ... & $\cdots$ & $\cdots$ & $\cdots$ & $\ldots$ \\
\hline • & $\cdots$ & $\cdots$ & $\cdots$ & $\cdots$ & $\cdots$ & $\cdots$ \\
\hline • & $\cdots$ & $\cdots$ & $\cdots$ & $\cdots$ & $\cdots$ & $\cdots$ \\
\hline c & $\mathrm{y}_{\mathrm{cl}}$ & $\dot{y}_{c 2}$ & . & $\mathrm{y}_{\mathrm{cb}}$ & $\mathrm{y}_{\mathrm{c}}$. & $\bar{y}_{c}$ \\
\hline
\end{tabular}

Total de Blocos

$\begin{array}{cccccc}\underset{\text { com tratamentos }}{\text { comuns }} & \mathrm{Y}_{.1} & \mathrm{Y}_{.2} & \ldots & \mathrm{Y}_{\text {.b }} & \mathrm{Y}\end{array}$

$\begin{array}{cccc}\mathrm{y}_{11} & \mathrm{y}_{21} & \cdots & \mathrm{y}_{\mathrm{bl}} \\ \mathrm{y}_{12} & \mathrm{y}_{22} & \cdots & \mathrm{y}_{\mathrm{b} 2} \\ \cdots & \cdots & \cdots & \cdots \\ \cdots & \cdots & \cdots & \cdots \\ \mathrm{y}_{1 \mathrm{r}_{1}} & \mathrm{y}_{2 \mathrm{r}_{2}} & \cdots & \mathrm{y}_{\mathrm{br}}\end{array}$

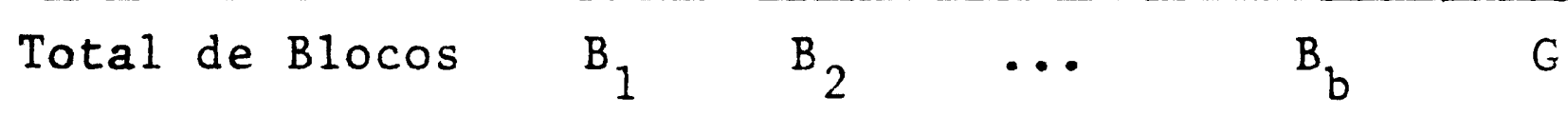

A análise de variância será efetuada da seguin te maneira. 


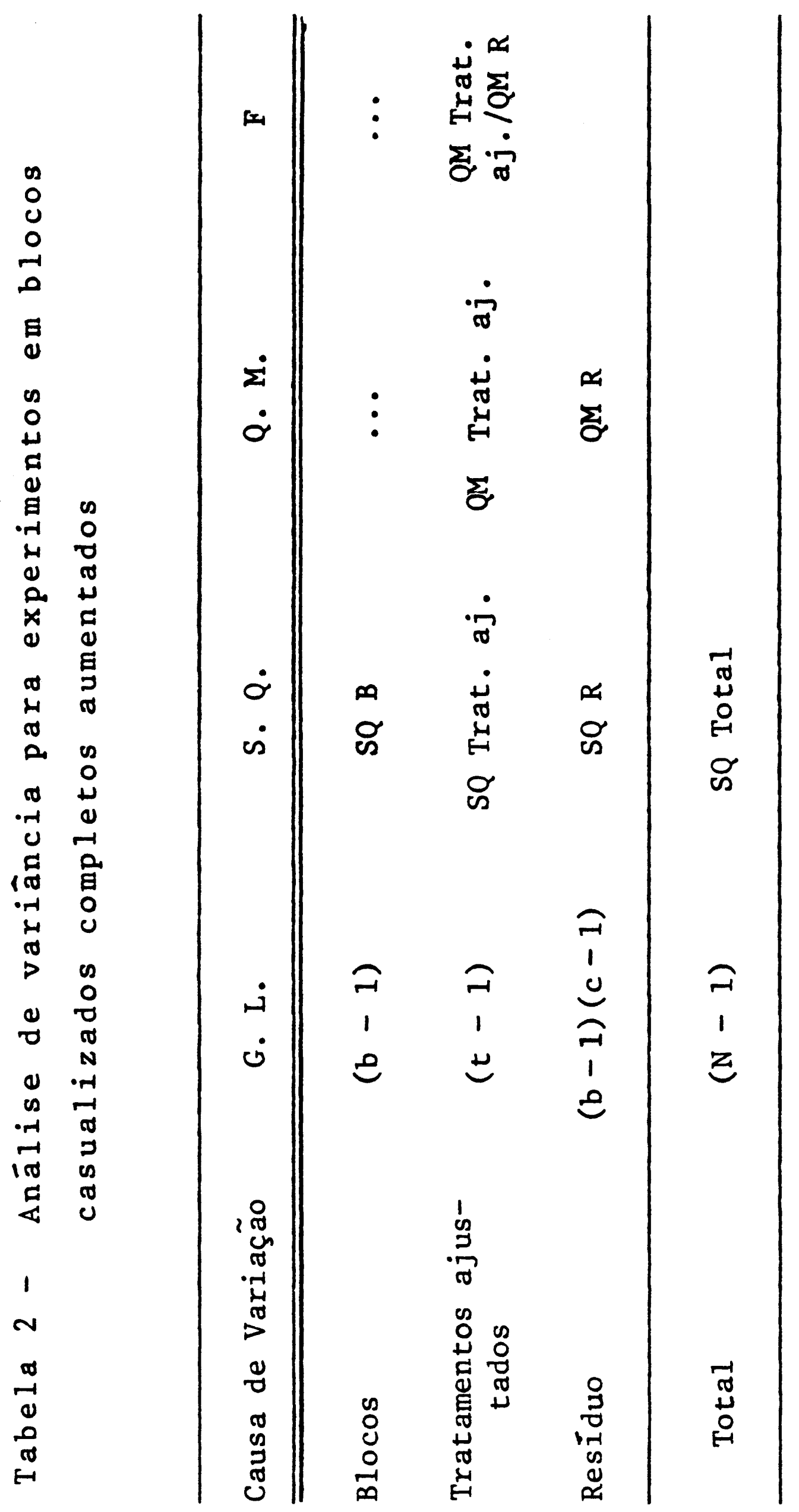


- cálculo para o número de graus de liberdade e soma de quadrados para o resíduo é efetuado atra vés dos tratamentos comuns, pois, ignorando os trā tamentos regulares, observa-se que eles se encontram em blocos casualizados. Procedendo o cálculo das somas de quadrados de maneira usual, teremos que:

$$
S Q R=\begin{aligned}
& S Q \text { Parcelas - SQ Blocos' - SQ Tratamentos } \\
& \text { comuns, }
\end{aligned}
$$

onde:

SQ Parcelas $=\sum_{i} \sum_{j} Y_{i j}^{2}-C^{\prime} \quad C^{\prime}=\frac{(Y . .)^{2}}{b . C}$

SQ Blocos' $=\frac{1}{C} \sum_{j} Y_{\cdot j}^{2}-C^{\prime}$

SQ Trat. comuns $=\frac{1}{b} \sum_{i} y_{i}^{2}-C^{\prime}$ 


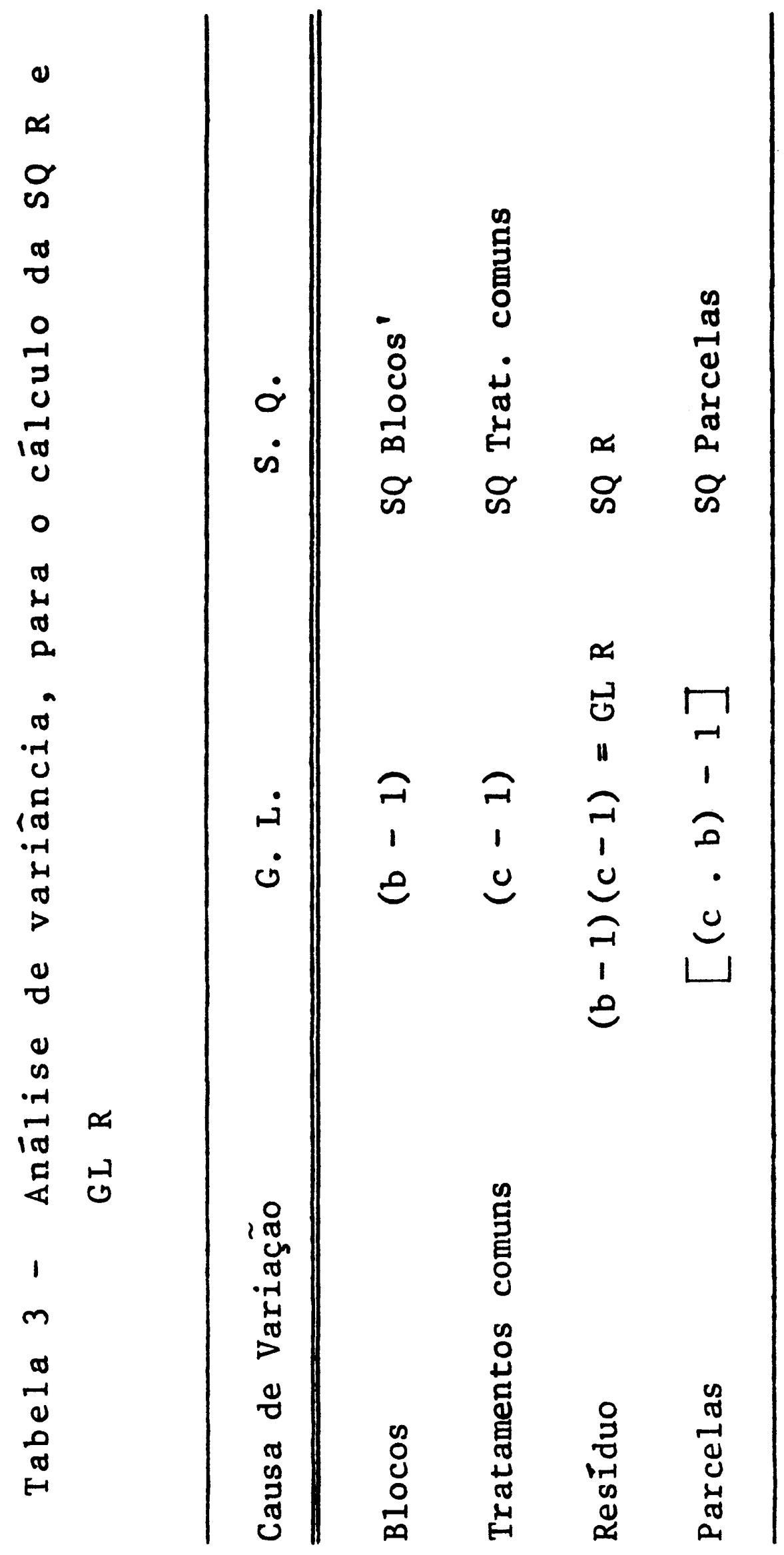


Voltamos ao cálculo da análise de variância pạ ra o delineamento em estudo:

$$
C=\frac{\mathrm{G}^{2}}{\mathrm{~N}}
$$

SQ Total $=\sum_{i} \sum_{j} y_{i j}^{2}+\sum_{j g} y_{j g}^{2}-C$

SQ $B=\frac{1}{N_{j}} \sum_{j} B_{j}^{2}-C$

SQ Trat. aj. = SQ Total - SQ B - SQ R

Se houver interesse em obter a significância dos efeitos para os blocos, outra alternativa da análise de variância para este delineamento será a da Tabela 4. 


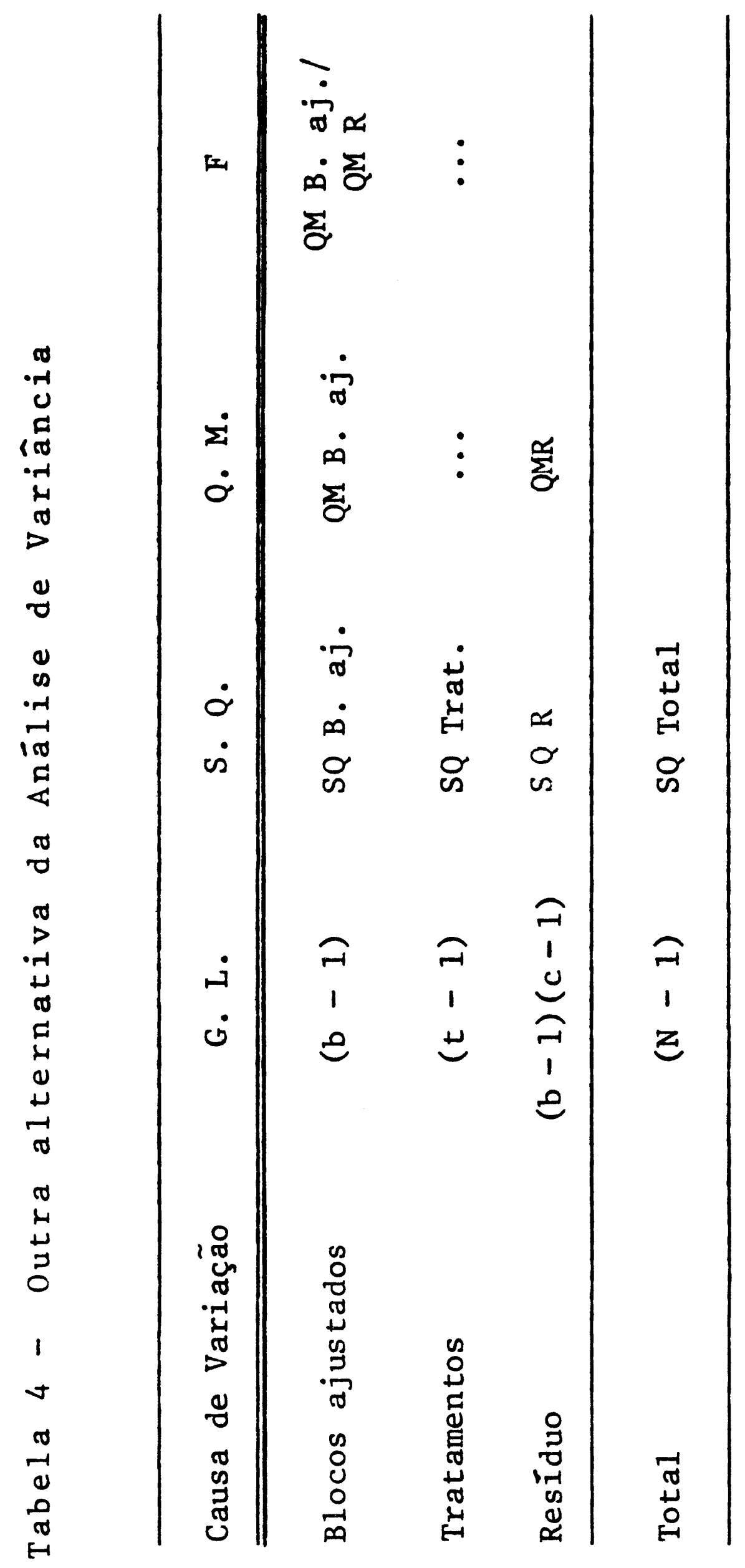


A soma de quadrados para o total e para o resí duo são as mesmas calculadas acima.

SQ Trat. $=\left[(1 / b) \sum_{i} Y_{i .}^{2}+\sum_{j g} \sum_{j g} y_{j g}\right]-C$

SQ B aj. = SQ Total - SQ Tratamento - SQ Residuo

$$
=\mathrm{SQ} \text { Blocos'. }
$$

As comparações das médias dos tratamentos pelo teste de Tukey segue as seguintes regras.

1. Entre dois tratamentos comuns:

$$
\begin{aligned}
\hat{V}\left(\bar{y}_{i}-\bar{y}_{i^{\prime}}\right) 2 \mathrm{QM} \mathrm{R} / \mathrm{b}, \quad \Delta & =\mathrm{q} \sqrt{(1 / 2) \hat{\mathrm{V}}\left(\bar{y}_{i}-\bar{y}_{i}{ }^{\prime}\right)} \\
& q(t, N \text { GL Res. })
\end{aligned}
$$

2. Entre os tratamentos regulares, devem-se con siderar dois casos:

a. Entre tratamentos regulares, dentro do mesmo bloco:

$$
\begin{aligned}
\hat{V}\left(y_{j g}^{\prime}-y_{j g^{\prime}}^{\prime}\right)=2 \text { QM R, } \Delta & =q \sqrt{(1 / 2) \hat{V}\left(y_{j g}^{\prime}-y_{j g^{\prime}}^{\prime}\right.} \\
& q(t, N \text { GL Res. })
\end{aligned}
$$

b. Entre tratamentos regulares, em blocos diferentes:

$$
\begin{aligned}
& \hat{V}\left(Y_{j g}^{\prime}-Y_{j^{\prime} g^{\prime}}^{\prime}\right)=2 \text { QM R }(I+1 / C), \\
& \Delta=q \sqrt{(1 / 2) \hat{V}\left(y_{j g}^{\prime}-y_{j}^{\prime} g^{\prime}\right)} \\
& q(t, N \text { GL Res.) }
\end{aligned}
$$


3. Entre tratamentos comuns e regulares:

$$
\begin{aligned}
\hat{v}\left(\bar{y}_{i}-y_{j g}^{\prime}\right)=Q M R(1+1 / c+1 / b+1 / b \cdot c), & , \\
\Delta & =q \sqrt{\left(1 / n^{\prime}\right) \hat{v}\left(\bar{y}_{1}-y_{j g}^{\prime}\right),}
\end{aligned}
$$

onde $n^{\prime}=b /(1+1 / b+1 / c+1 / b \cdot c)$,

$q(t, N$ GL Res.)

As estimativas das médias dos tratamentos são obtidas da seguinte maneira:

1. Para os tratamentos comuns: Não há necessidade de efetuar o ajuste, por estarem repetidos $b$ ve zes. Calcula-se simplesmente a média aritméti= ca dos dados respectivos. Portanto:

$\bar{y}_{i}=y_{i} / \mathrm{b}$

2. Para os tratamentos regulares: há necessidade de efetuar o ajuste dos dados respectivos, através da subtração de uma correção $A_{j}(j=, 1, \ldots, b)$ que se calcula da seguinte forma:

$$
A_{j}=Y \cdot j / C-Y . \cdot / C \cdot b, \quad \sum_{j} A_{j}=0 .
$$

Portanto o tratamento regular ajustado será:

$$
y_{j g}^{\prime}=Y_{j g}-A_{j} \text {. }
$$

Para melhor ilustrar a aplicação do método pro posto, suponhamos hipoteticamente como exemplo um experimento de competição de "variedades" de cana-de-açúcar, com as seguintes características:

- Número total de tratamentos, $t=15$ "varieda des" de cana-de-açúcar;

- Número de tratamentos comuns, $c=3$ "varieda des" de cana-de-açúcar; indicadas por A, B $\bar{e}$ C ; 
- Número de tratamentos regulares, $z=12$ "variedades" de cana-de-açưcar, indicadas por $d$, $e, f, g, h, i, j, k, l, m, n, o$;

- Número de blocos, $\mathrm{b}=4$;

- Número de parcelas por bloco, $\mathrm{N}_{j}=6$;

- Número de tratamentos regulares por bloco, $r_{j}=3 ;$

- Numeto total de parcelas, $\mathrm{N}=24$.

Tabela 5 - Dados hipotéticos em t/ha.

\begin{tabular}{|c|c|c|c|c|c|}
\hline \multirow{2}{*}{ " Variedades" } & \multicolumn{4}{|c|}{ Blocos } & \multirow{2}{*}{ Total } \\
\hline & 1 & 2 & 3 & 4 & \\
\hline A & 128 & 120 & 130 & 116 & 494 \\
\hline B & 110 & 112 & 119 & 97 & 438 \\
\hline $\mathrm{C}$ & 126 & 131 & 145 & 135 & 537 \\
\hline Total de Blocos com & 364 & 363 & 394 & 348 & 1.469 \\
\hline $\begin{array}{l}\text { Iratamentos comuns } \\
\text { d }\end{array}$ & 129 & & & & 129 \\
\hline $\mathrm{e}$ & 112 & & & & 112 \\
\hline $\mathrm{f}$ & 156 & & & & 156 \\
\hline g & & 129 & & & 129 \\
\hline $\mathrm{h}$ & & 154 & & & 154 \\
\hline $\mathbf{i}$ & & 165 & & & 165 \\
\hline $\mathbf{j}$ & & & 131 & & 131 \\
\hline k & & & 136 & & 136 \\
\hline$\ell$ & & & 126 & & 126 \\
\hline m & & & & 111 & 111 \\
\hline $\mathbf{n}$ & & & & 131 & 131 \\
\hline o & & & & 134 & 134 \\
\hline Total de Blocos & 761 & 811 & 787 & 724 & 3.083 \\
\hline
\end{tabular}


- Cálculo da Soma de Quadrados para o Resíduo

Consideram-se,para tal, somente os dados referentes aos tratamentos comuns.

SQ R = SQ Parcelas - SQ Tratamentos comuns - SQ Blocos',

onde teremos, que:

SQ Parcelas $=\left(128^{2}+\ldots+135^{2}\right)-1.469^{2} / 12=$

$$
=1.810,92
$$

SQ Blocos' $=(1 / 3)\left(364^{2}+\ldots+348^{2}\right)-1.469^{2} / 12=$

$$
=371,59
$$

SQ Trat. comuns $=(1 / 4)\left(494^{2}+\ldots+537^{2}\right)-1.469^{2} / 12=$

$$
=1.232,17
$$

SQ Resíduo $=1.810,92-1.232,17-371,59=207,16$

- Cálculo da Análise de Variância

SQ Total $=\left(128^{2}+\ldots+134^{2}\right)-3.082^{2} / 24$

$$
=5.677,96
$$

SQ Blocos $=(1 / 6)\left(761^{2}+\ldots+724^{2}\right)-3.0822 / 24=$

$$
=694,13
$$

SQ Trat. aj . = 5.677,96-694,13-207,16=

$$
=4.776,67
$$




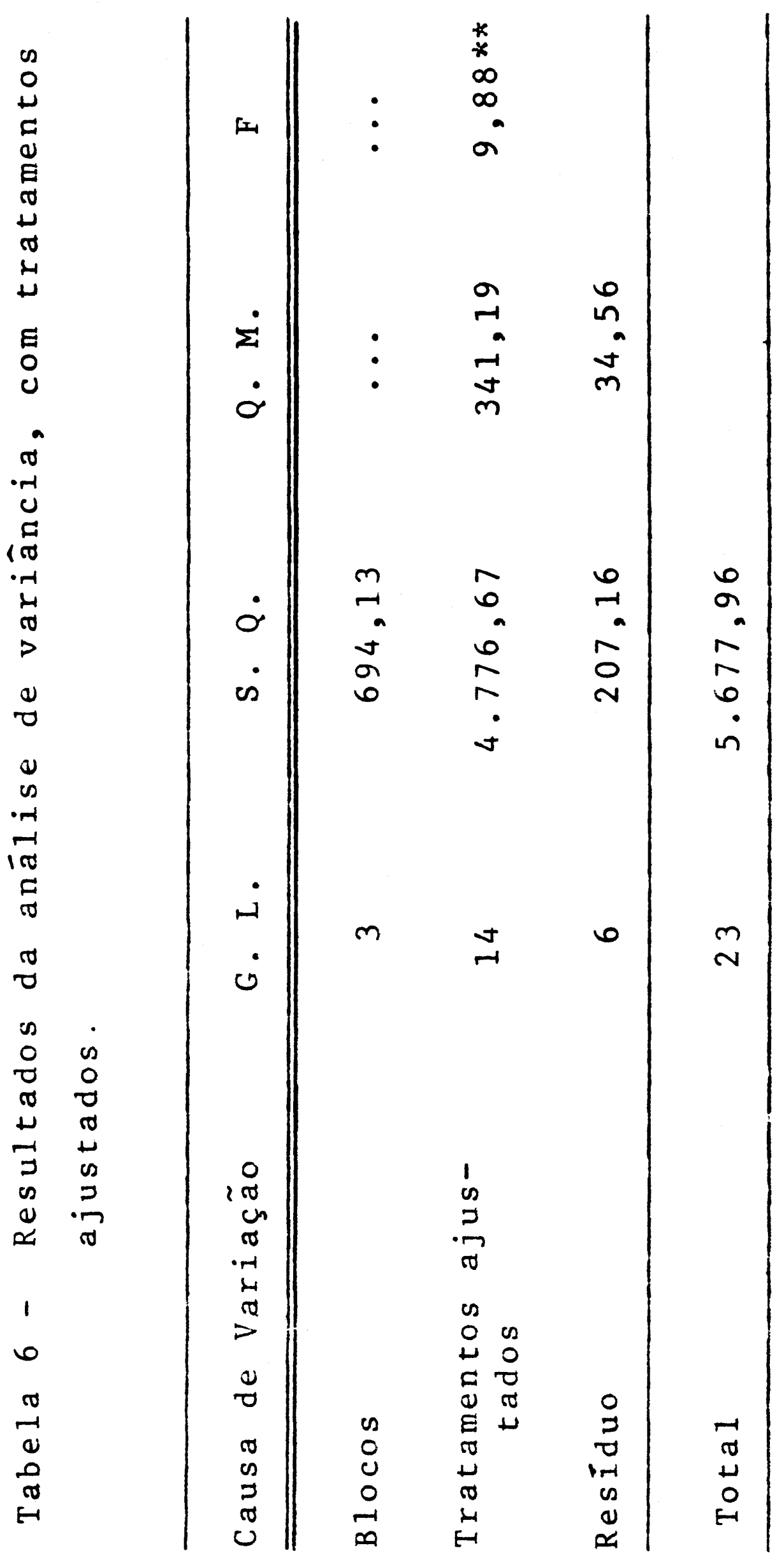


Outra alternativa da análise de variância para este delineamento será a sequinte:

Tabela 7. Resultado da anālise de variância, com blocos ajus tados.

\begin{tabular}{|c|c|c|c|c|}
\hline Causa de Variação & G.L. & S.Q. & Q.M. & $\mathbf{F}$ \\
\hline $\begin{array}{l}\text { Blocos ajustados } \\
\text { Tratamentos } \\
\text { Resíduo }\end{array}$ & $\begin{array}{r}3 \\
14 \\
6\end{array}$ & $\begin{array}{r}371,59 \\
5.099,21 \\
207,16\end{array}$ & $\begin{array}{c}123,86 \\
3 \ddot{4}, 56\end{array}$ & $\begin{array}{r}3,59 \\
\ldots\end{array}$ \\
\hline Total & 23 & $5.677,96$ & & \\
\hline
\end{tabular}

SQ Trat. $=(1 / 4)\left(494^{2}+438^{2}+537^{2}\right)+131^{2}+\ldots+$ $+134^{2}-3082^{2} / 24=5.099,21$

SQ Blocos aj. $=5.677,96-207,16-5.099,21=$

$$
=371,59
$$

Como podemos observar :

SQ Blocos aj. = SQ Blocos' $=371,59$.

- Estimativa das Médias dos Tratamentos

1. Para os tratamentos comuns:

$$
\begin{aligned}
& \bar{y}_{A}=494 / 4=123,40 \mathrm{t} / \mathrm{ha} \\
& \bar{y}_{B}=190,50 \mathrm{t} / \mathrm{ha} \\
& \bar{y}_{C}=134,25 \mathrm{t} / \mathrm{ha}
\end{aligned}
$$


2. Para os tratamentos regulares: Cálculo das correções para o ajuste das produções:

$$
\begin{aligned}
& A_{j}=Y \cdot j / c-Y . . / C \cdot b \quad(j=1, \ldots, b) \\
& A_{1}=364 / 3-1.469 / 12=-1,08 \\
& A_{2}=363 / 3-1.469 / 12=-1,42 \\
& A_{3}=394 / 3-1.469 / 12=8,92 \\
& A_{4}=348 / 3-1.469 / 12=-6,42
\end{aligned}
$$

As produções ajustadas para os tratamentos regulares são as seguintes:

$$
\begin{aligned}
& y_{j g}^{\prime}=y_{j g}-A_{j} \\
& y_{d}^{\prime}=y_{11}^{\prime}=129+1,08=130,08 \mathrm{t} / \mathrm{ha} \\
& y_{e}^{\prime}=y_{12}^{\prime}=112+1,08=113,08 \mathrm{t} / \mathrm{ha} \\
& y_{f}^{\prime}=y_{13}^{\prime}=156+1,08=157,08 \mathrm{t} / \mathrm{ha} \\
& y_{g}^{\prime}=y_{21}^{\prime}=129+1,42=130,42 \mathrm{t} / \mathrm{ha} \\
& y_{h}^{\prime}=y_{22}^{\prime}=154+1,42=155,42 \mathrm{t} / \mathrm{ha} \\
& y_{1}^{\prime}=y_{23}^{\prime}=165+1,42=166,42 \mathrm{t} / \mathrm{ha} \\
& y_{j}^{\prime}=y_{31}^{\prime}=131-8,92=122,08 \mathrm{t} / \mathrm{ha} \\
& y_{k}^{\prime}=y_{32}^{\prime}=136-8,92=127,08 \mathrm{t} / \mathrm{ha} \\
& y_{2}^{\prime}=y_{33}^{\prime}=126-8,92=117,08 \mathrm{t} / \mathrm{ha} \\
& y_{m}^{\prime}=y_{41}^{\prime}=111+6,42=117,42 \mathrm{t} / \mathrm{ha} \\
& y_{n}^{\prime}=y_{42}^{\prime}=313+6,42=137,42 \mathrm{t} / \mathrm{ha} \\
& y_{0}^{\prime}=y_{43}^{\prime}=134+6,42=140,42 \mathrm{t} / \mathrm{ha}
\end{aligned}
$$


SUMMARY

\section{AUGMENTED DESIGNS IN COMPLETE RANDOMIZED BLOCKS}

This paper object is to show an easier methodology for the variance analysis of experiments in augmented complete randomized blocks.

\section{LITERATURA CITADA}

FEDERER, W.T., 1956. Augmented designs. Hawaiian Planter's Record 55:191-208.

PIMENTEL GOMES, F.; GUIMARÃES, R.F., 1958. Joint analysis of experiments in complete randomised blocks with some common treatments. Biometrics, 14: 521-526.

PIMENTEL GOMES, F., 1978. Curso de Estatistica Experimentah, 8a. Edição, Livraria Nobel, São Paulo. 
